Supplement of Biogeosciences, 19, 541-558, 2022

https://doi.org/10.5194/bg-19-541-2022-supplement

(C) Author(s) 2022. CC BY 4.0 License.

(c) (i)

Supplement of

\title{
Resolving temperature limitation on spring productivity in an evergreen conifer forest using a model-data fusion framework
}

Stephanie G. Stettz et al.

Correspondence to: Stephanie G. Stettz (sstettz@uci.edu)

The copyright of individual parts of the supplement might differ from the article licence. 

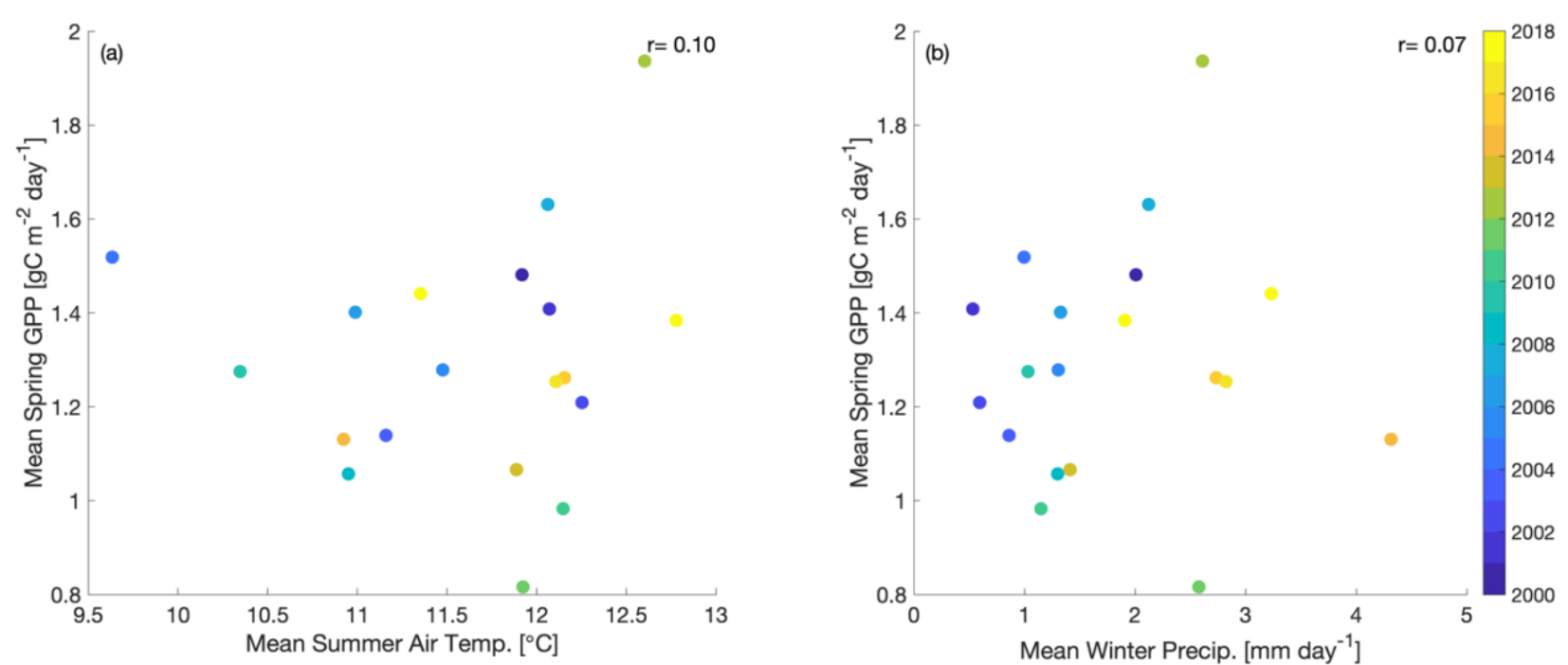

Figure S1. Scatter plots showing the relationship between observed a.) mean summer (June-September) air temperature and b.) mean winter (December-February) precipitation and mean spring (March-May) GPP at Niwot Ridge (US-NR1). Dot color varies by year (2000-2018).'r' is the Pearson's linear correlation coefficient.

Figure S2. Timeseries of mean annual GPP derived from the AmeriFlux Niwot Ridge eddy covariance tower (US-NR1) from 51 2000-2018.

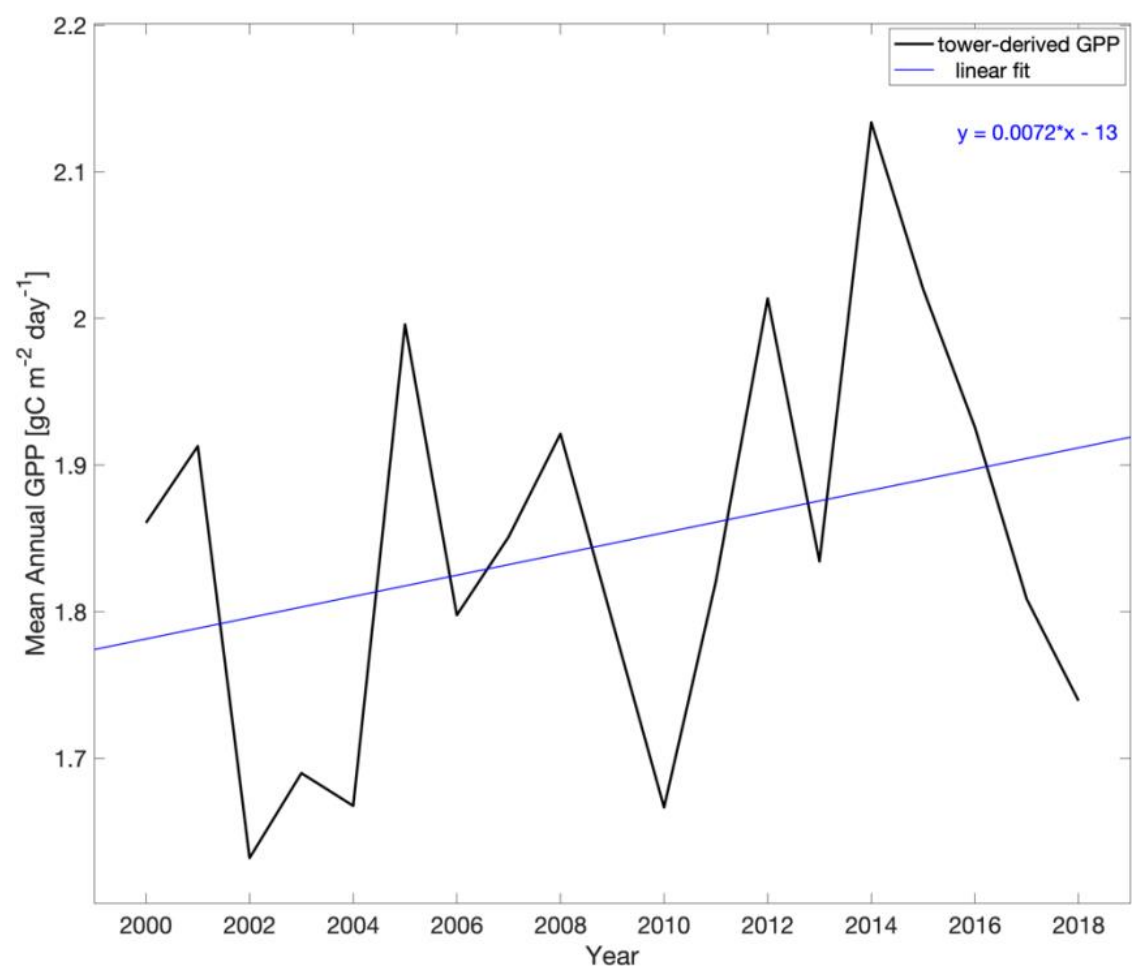



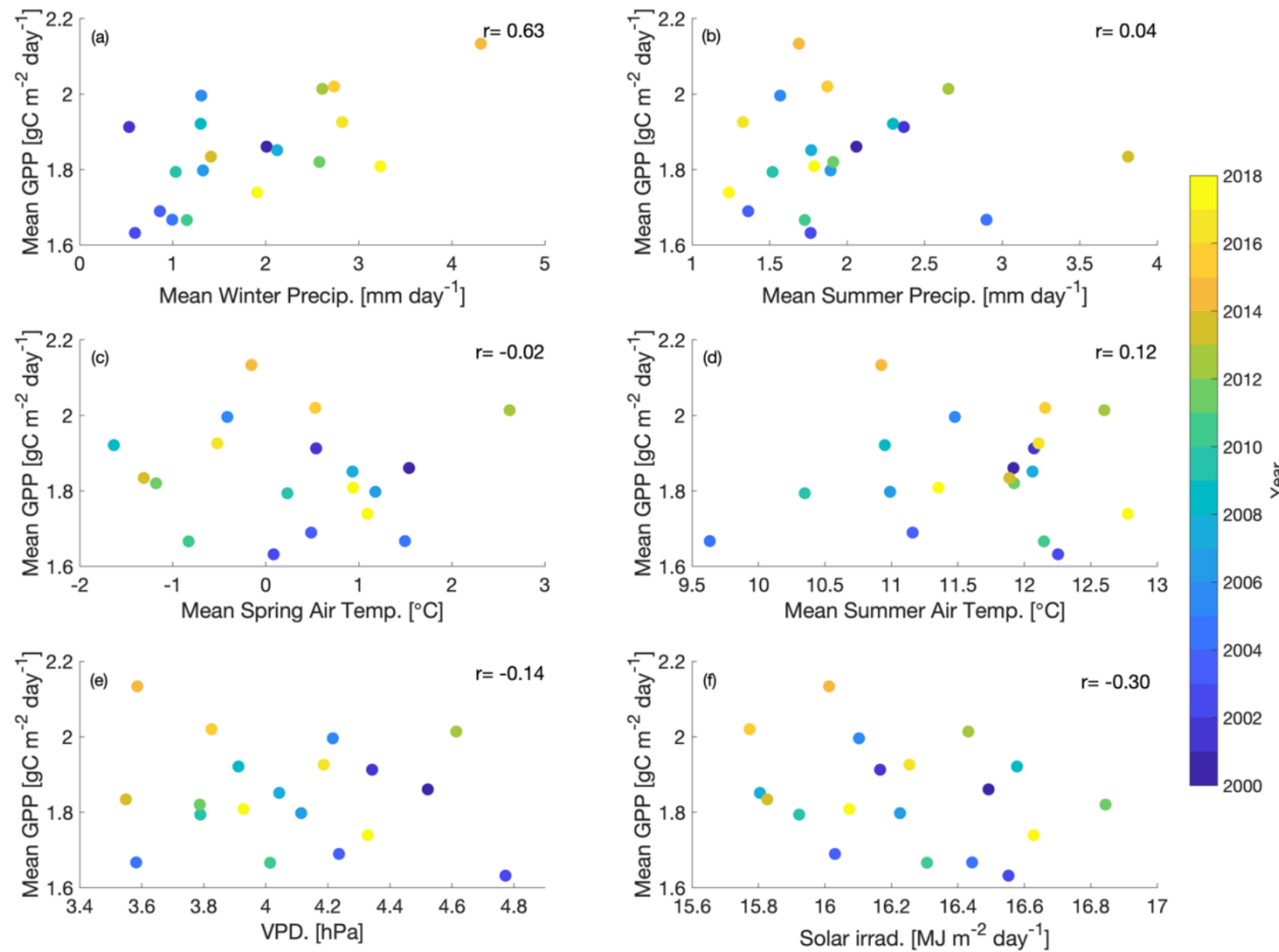

Figure S3. Scatter plots showing the relationship between observed meteorological data (a.) mean annual winter (Dec-Feb) precipitation, b.) mean summer (Jun-Sep) precipitation, c.) mean spring (Mar-May) air temperature, d.)mean summer (Jun-Sep) air temperature, e.) mean annual vapor pressure deficit (VPD) and f.) mean annual shortwave irradiance, and mean annual GPP at Niwot Ridge (US-NR1). Dot color varies by year (2000-2018).'r' is the Pearson's linear correlation coefficient. 

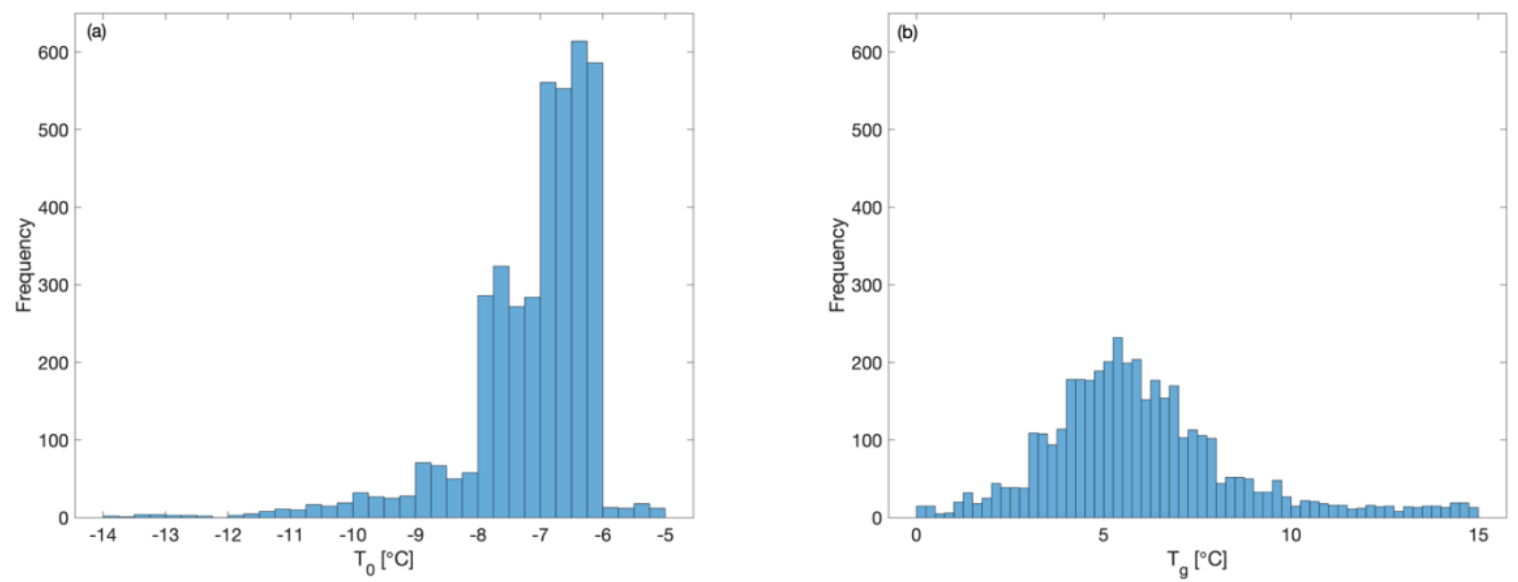

Figure S4. Histograms of a.) $\mathrm{T}_{0}$ (photosynthesis shutdown air temperature) and $\mathrm{b}$.) $\mathrm{T}_{\mathrm{g}}$ (photosynthesis initiation air temperature) parameters included in CARDcold experiments $(\mathrm{N}=4000$ ensembles).

73
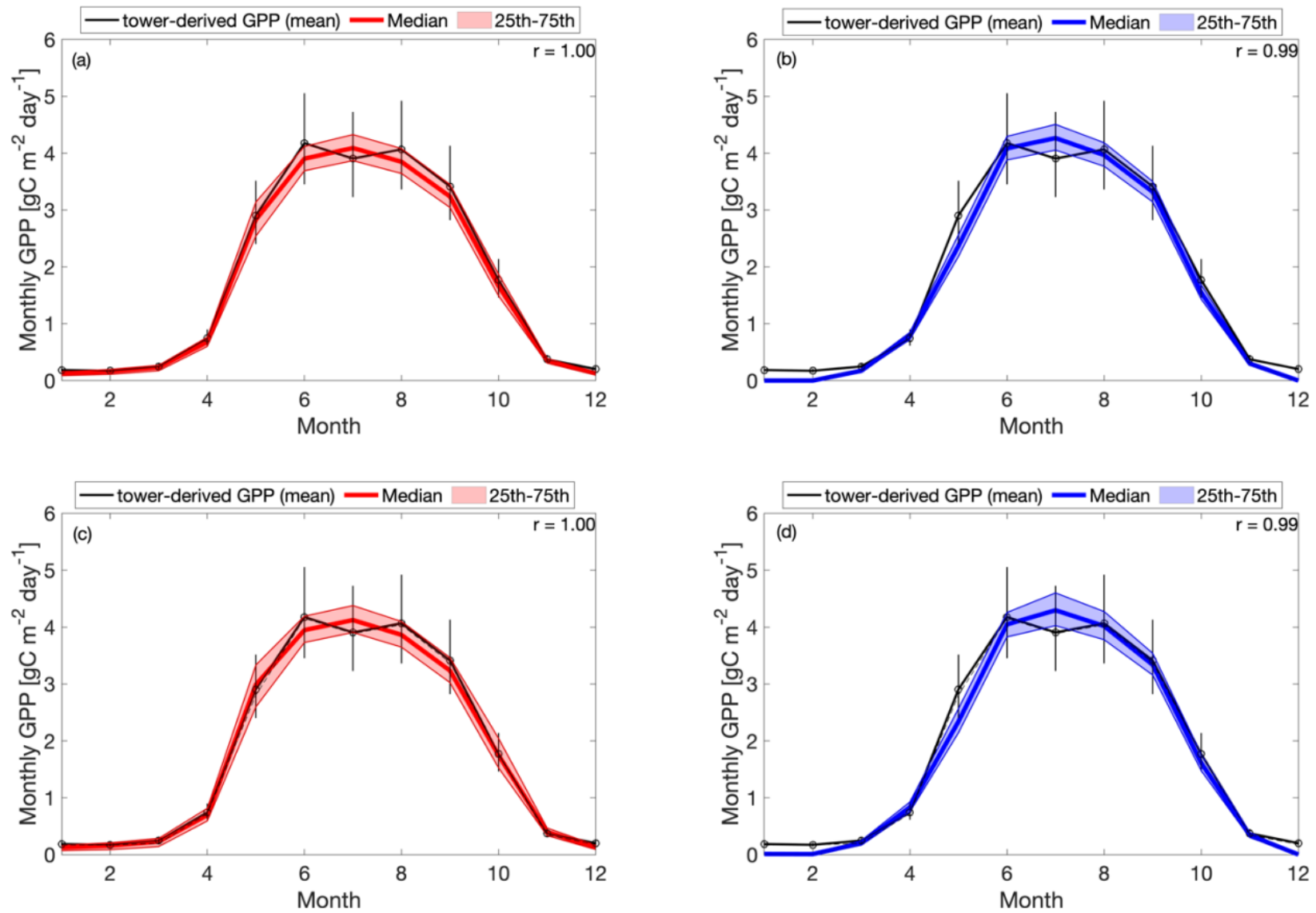

Figure S5. Tower-derived (black line) mean monthly GPP with simulated seasonal cycles at US-NR1 for 2000-2018, for a.) CARD, b.) CARDcold, c.) CARD-Half and d.) CARDcold-Half experiments. Model outputs include the median value of each experiment (bold color line) with the $25^{\text {th }}-75$ th percentiles of the ensembles (shaded area). The median is plotted instead of the mean to avoid impact of outlier ensemble members $(\mathrm{N}=4000$ members $)$. Error bars $=$ tower-derived GPP multiplied/divided by $\exp \left(\operatorname{sqrt}\left(\log (2)^{\wedge} 2^{*} \mathrm{n}\right) / \mathrm{n}\right), \mathrm{n}=\#$ of years in average $(\mathrm{n}=19)$. ' $\mathrm{r}$ ' is Pearson's $r$ coefficient. Experiments are described in more detail in Table 1. 

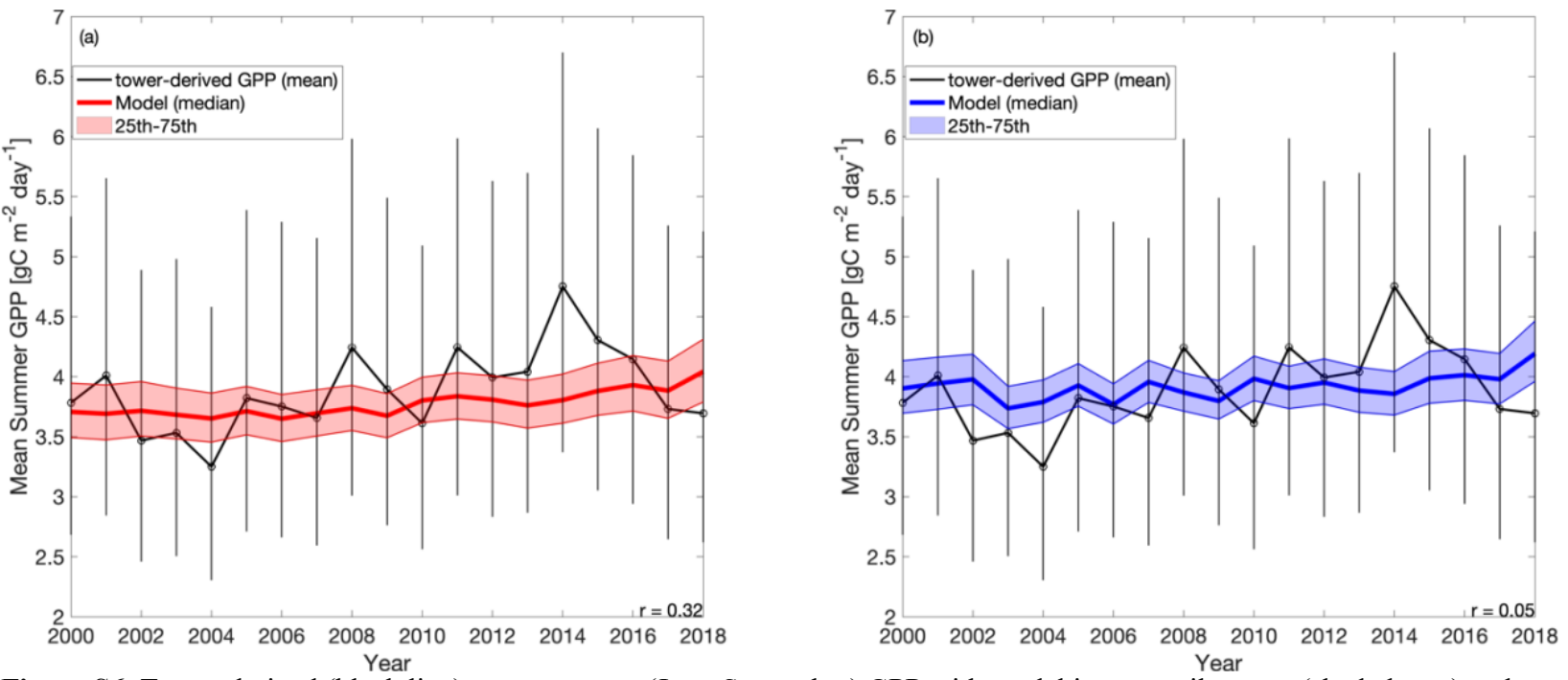

Figure S6. Tower-derived (black line) mean summer (June-September) GPP with model interquartile range (shaded area) and median (bold color line) of summer GPP outputs for a.) CARD and b.) CARDcold experiments. Error bars = tower-derived GPP multiplied/divided by $\exp \left(\operatorname{sqrt}\left(\log (2)^{\wedge} 2^{*} \mathrm{n}\right) / \mathrm{n}\right), \mathrm{n}=\#$ of months in average $(\mathrm{n}=4)$.
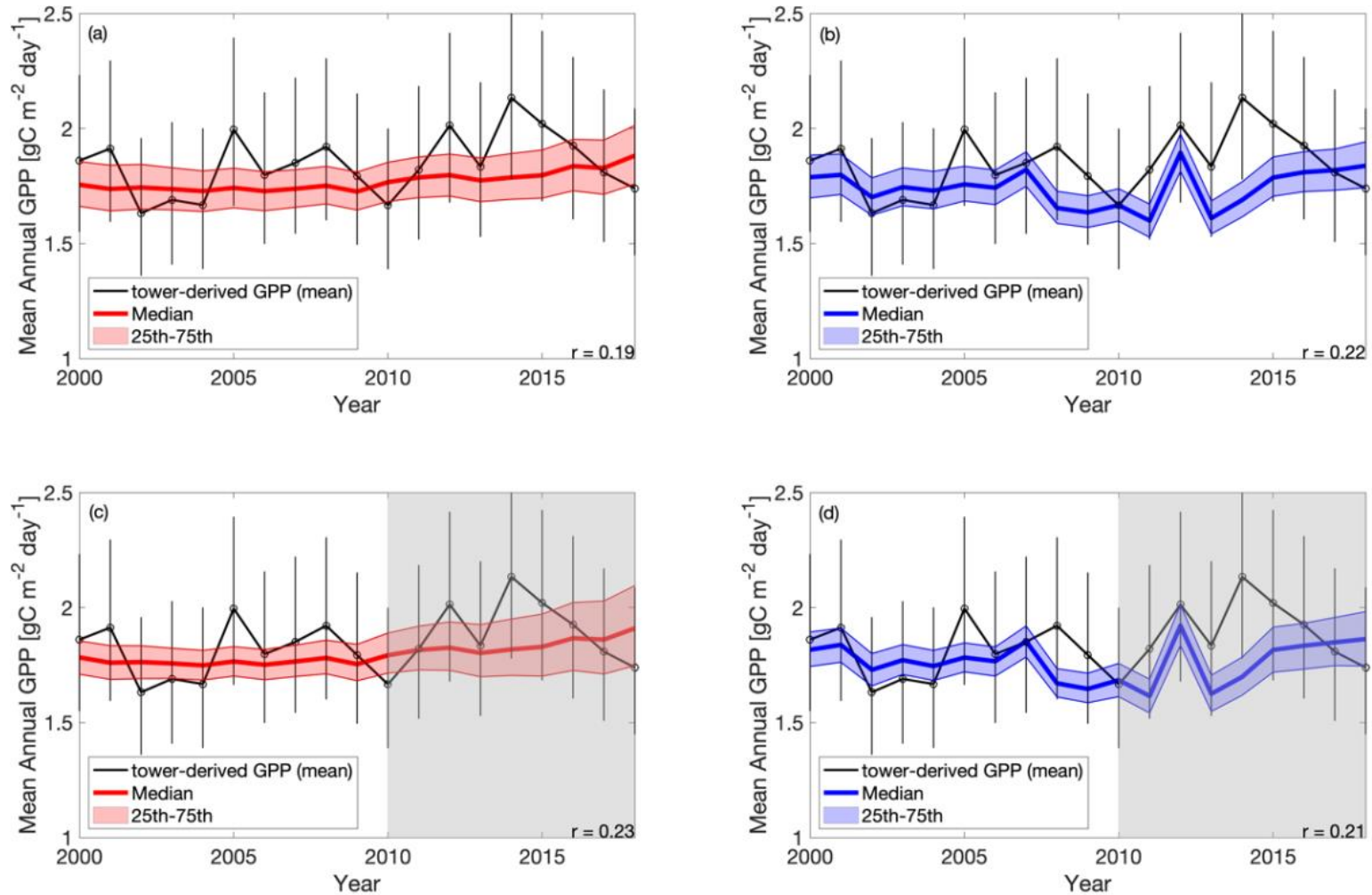

89

91

92

93

94

Figure S7. Tower-derived (black line) mean annual GPP with model interquartile range (color shaded area) and median (bold color line) of annual GPP outputs for a.) CARD, b.) CARDcold, c.) CARD-Half, and d.) CARDcold-Half experiments. The grey regions indicate no data assimilation (i.e. testing window). Error bars $=$ tower-derived GPP multiplied/divided by $\exp \left(\operatorname{sqrt}\left(\log (2)^{\wedge} 2 * n\right) / n\right), n=\#$ of years in average $(n=19)$. 

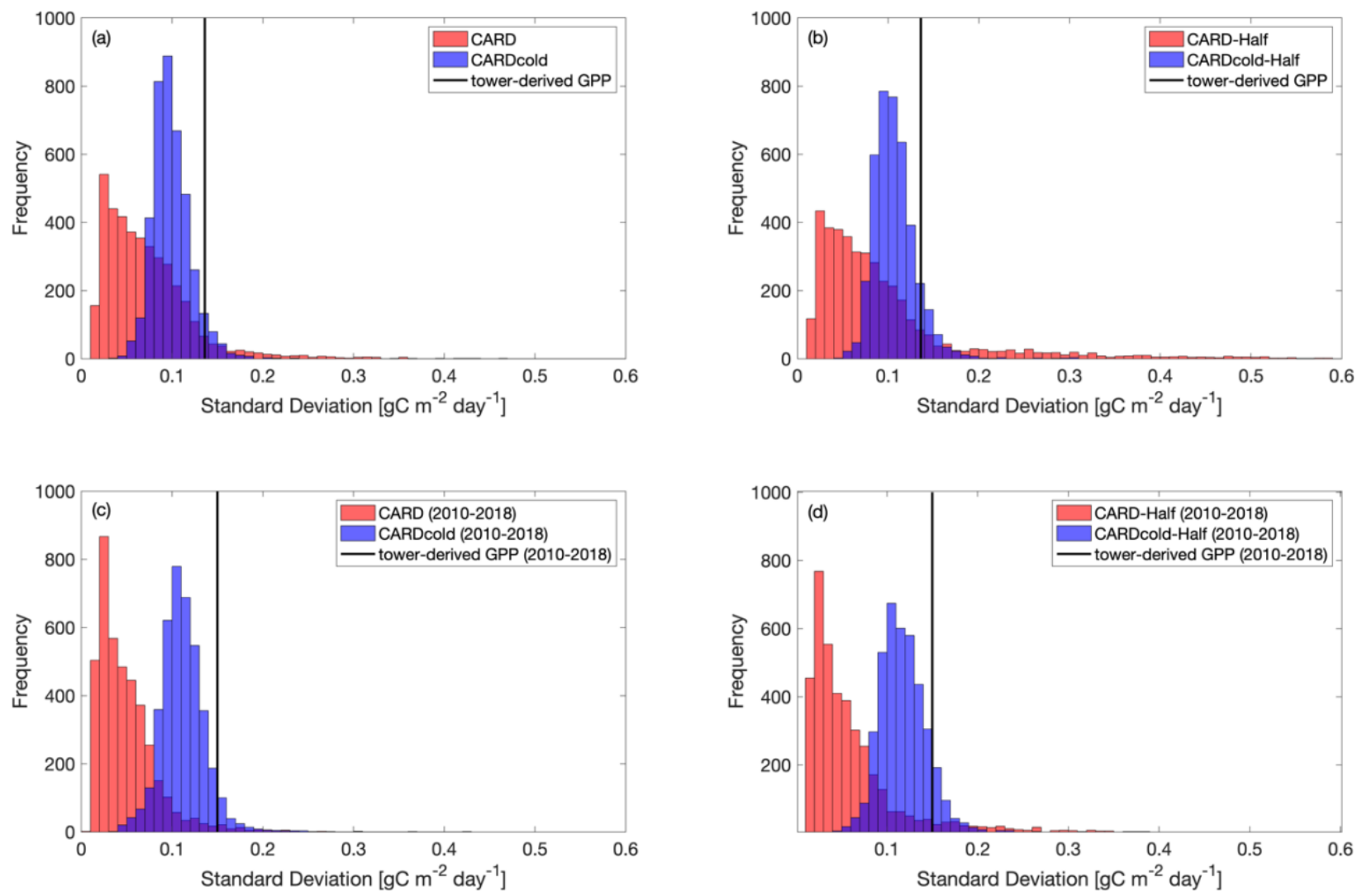

Figure S8. Histograms comparing standard deviation in mean annual GPP across all ensembles (N=4000) for CARD (red bars) and CARDcold (blue bars) experiments with a.) full assimilation, b.) half assimilation, c.) full assimilation for the second decade (2010-2018), and d.) half assimilation for the second decade (2010-2018). Black line indicates standard deviation in towerderived mean annual GPP ( $\mathrm{std}=0.14 \mathrm{gC} \mathrm{m}^{-2}$ day $^{-1}$ for full period $(\mathrm{a}-\mathrm{b})$, $\mathrm{std}=0.15 \mathrm{gC} \mathrm{m}^{-2} \mathrm{day}^{-1}$ for 2010-2018 (c-d)). 


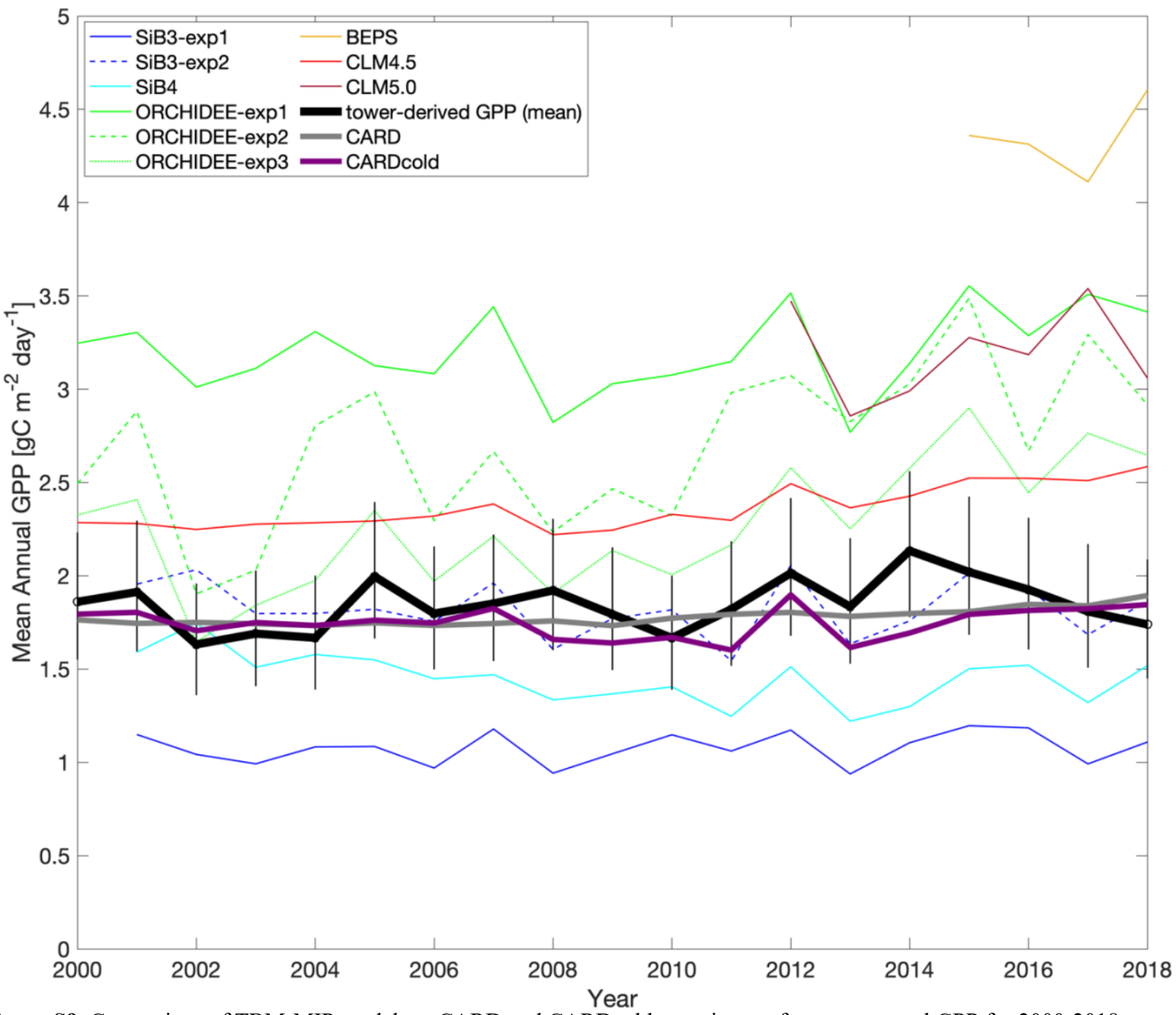

Figure S9. Comparison of TBM-MIP models to CARD and CARDcold experiments for mean annual GPP for 2000-2018.

116

117

118

119

120

121

122

123

124

125

126

127

128

129

130

131

132

133

134

135

136 
Table S1. Pearson's linear r, R-squared, p-value, standard deviation, root mean square error (RMSE), and mean bias error (MBE) for TBM-MIP and all CARDAMOM experiments to Niwot Ridge tower-derived mean spring GPP for 2015-2018. All relevant statistics calculated at 5\% significance level.

140

141

142

143

144
145

\begin{tabular}{|l|l|l|l|l|l|l|}
\hline model & r-value & $\begin{array}{l}\text { R- } \\
\text { squared }\end{array}$ & $\begin{array}{l}\mathrm{p} \text {-value }(\alpha \\
=0.05)\end{array}$ & $\begin{array}{l}\mathrm{RMSE} \\
\left(\mathrm{gC} \mathrm{m}^{-2} \mathrm{~d}^{-}\right. \\
1\end{array}$ & $\begin{array}{l}\mathrm{MBE}(\mathrm{gC} \\
\left.\mathrm{m}^{-2} \mathrm{~d}^{-1}\right)\end{array}$ & $\begin{array}{l}\text { standard } \\
\text { deviation }(\mathrm{gC} \\
\left.\mathrm{m}^{-2} \mathrm{~d}^{-1}\right)\end{array}$ \\
\hline CARD-Half & 0.82 & 0.67 & 0.18 & 0.08 & 0.06 & 0.05 \\
CARD & 0.82 & 0.67 & 0.18 & 0.13 & 0.12 & 0.04 \\
CARDcold-Half & 0.78 & 0.61 & 0.22 & 0.22 & 0.20 & 0.18 \\
CARDcold & 0.76 & 0.58 & 0.24 & 0.25 & 0.22 & 0.19 \\
SiB3-exp1 & 0.44 & 0.20 & 0.56 & 1.24 & 1.24 & 0.08 \\
SiB3-exp2 & 0.42 & 0.17 & 0.58 & 1.16 & 1.16 & 0.09 \\
SiB4 & 0.57 & 0.33 & 0.43 & 1.01 & 1.01 & 0.06 \\
ORCHIDEE-exp1 & 0.67 & 0.45 & 0.33 & 1.43 & -1.42 & 0.19 \\
ORCHIDEE-exp2 & -0.09 & 0.01 & 0.91 & 1.38 & -1.37 & 0.20 \\
ORCHIDEE-exp3 & -0.26 & 0.07 & 0.74 & 0.93 & -0.92 & 0.08 \\
BEPS & 0.84 & 0.70 & 0.16 & 2.46 & -2.46 & 0.16 \\
CLM4.5 & 0.74 & 0.55 & 0.26 & 0.36 & -0.35 & 0.15 \\
CLM5.0 & 0.91 & 0.82 & 0.09 & 1.10 & -1.09 & 0.24 \\
\hline
\end{tabular}

Table S2. Pearson's linear r, R-squared, p-value, standard deviation, root mean square error (RMSE), and mean bias error (MBE) for TBM-MIP and all CARDAMOM experiments to Niwot Ridge tower-derived mean annual GPP for 2001-2018. All relevant statistics calculated at 5\% significance level.

\begin{tabular}{|l|l|l|l|l|l|l|}
\hline model & r-value & R-squared & $\begin{array}{l}\mathrm{p} \text {-value } \\
(\alpha=0.05)\end{array}$ & $\begin{array}{l}\text { RMSE } \\
\left(\mathrm{gC} \mathrm{m}^{-2} \mathrm{~d}^{-}\right.\end{array}$ & $\begin{array}{l}\text { MBE }(\mathrm{gC} \\
\left.\mathrm{m}^{-2} \mathrm{~d}^{-1}\right)\end{array}$ & $\begin{array}{l}\text { standard } \\
\text { deviation }\left(\mathrm{gC} \mathrm{m}^{-}\right. \\
\left.2 \mathrm{~d}^{-1}\right)\end{array}$ \\
\hline CARD-Half & 0.23 & 0.05 & 0.35 & 0.14 & 0.05 & 0.05 \\
CARD & 0.19 & 0.04 & 0.44 & 0.15 & 0.07 & 0.04 \\
CARDcold-Half & 0.21 & 0.04 & 0.41 & 0.17 & 0.08 & 0.09 \\
CARDcold & 0.22 & 0.05 & 0.37 & 0.18 & 0.11 & 0.08 \\
SiB3-exp1 & 0.32 & 0.10 & 0.19 & 0.78 & 0.77 & 0.09 \\
SiB3-exp2 & 0.10 & 0.01 & 0.69 & 0.19 & 0.02 & 0.15 \\
SiB4 & -0.25 & 0.06 & 0.32 & 0.45 & 0.39 & 0.14 \\
ORCHIDEE-exp1 & 0.21 & 0.04 & 0.40 & 1.38 & -1.36 & 0.23 \\
ORCHIDEE-exp2 & 0.60 & 0.36 & 0.01 & 0.94 & -0.87 & 0.43 \\
ORCHIDEE-exp3 & 0.64 & 0.41 & 0.00 & 0.50 & -0.42 & 0.34 \\
BEPS & -0.25 & 0.06 & 0.75 & 2.48 & -2.47 & 0.20 \\
CLM4.5 & 0.34 & 0.12 & 0.17 & 0.54 & -0.52 & 0.11 \\
CLM5.0 & 0.03 & 0.00 & 0.96 & 1.30 & -1.27 & 0.25 \\
\hline
\end{tabular}


156

159

Table S3. Pearson's linear r, R-squared, p-value, standard deviation, root mean square error (RMSE), and mean bias error (MBE) for TBM-MIP and all CARDAMOM experiments to Niwot Ridge tower-derived mean monthly GPP for 2015-2018. All relevant statistics calculated at 5\% significance level.

\begin{tabular}{|l|l|l|l|l|l|l|}
\hline model & r-value & R-squared & $\begin{array}{l}\mathrm{p} \text {-value }(\alpha= \\
0.05)\end{array}$ & $\begin{array}{l}\text { RMSE }(\mathrm{gC} \\
\left.\mathrm{m}^{-2} \mathrm{~d}^{-1}\right)\end{array}$ & $\begin{array}{l}\mathrm{MBE}(\mathrm{gC} \\
\left.\mathrm{m}^{-2} \mathrm{~d}^{-1}\right)\end{array}$ & $\begin{array}{l}\text { standard } \\
\text { deviation }\left(\mathrm{gC} \mathrm{m}^{-2}\right. \\
\left.\mathrm{d}^{-1}\right)\end{array}$ \\
\hline CARD-Half & 1.00 & 1.00 & 0.00 & 0.09 & 0.007 & 1.76 \\
CARD & 1.00 & 1.00 & 0.00 & 0.09 & 0.04 & 1.75 \\
CARDcold-Half & 0.99 & 0.98 & 0.00 & 0.23 & 0.04 & 1.79 \\
CARDcold & 0.99 & 0.98 & 0.00 & 0.22 & 0.07 & 1.80 \\
SiB3-exp1 & 0.91 & 0.84 & 0.00 & 1.02 & 0.76 & 1.50 \\
SiB3-exp2 & 0.92 & 0.84 & 0.00 & 1.06 & 0.01 & 2.47 \\
SiB4 & 0.94 & 0.88 & 0.00 & 0.73 & 0.42 & 1.77 \\
ORCHIDEE-exp1 & 0.98 & 0.95 & 0.00 & 1.80 & -1.55 & 2.58 \\
ORCHIDEE-exp2 & 0.95 & 0.91 & 0.00 & 1.48 & -1.20 & 2.40 \\
ORCHIDEE-exp3 & 0.96 & 0.93 & 0.00 & 0.95 & -0.81 & 1.90 \\
BEPS & 0.98 & 0.95 & 0.00 & 2.56 & -2.46 & 2.32 \\
CLM4.5 & 0.99 & 0.98 & 0.00 & 1.20 & -0.65 & 2.76 \\
CLM5.0 & 0.97 & 0.95 & 0.00 & 1.60 & -1.38 & 2.45 \\
\hline
\end{tabular}

